\title{
Using Dental Age to Estimate Chronological Age in Czech Children Aged 3-18 Years
}

\author{
Kristina Ginzelová1, Tat'jana Dostálová1, Hana Eliášová ${ }^{2}$, \\ Alex Vinšů ', Antonín Buček', Michaela Bučková1 \\ ${ }^{1}$ Department of Stomatology, Second Faculty of Medicine, Charles University \\ in Prague and University Hospital Motol, Prague, Czech Republic; \\ ${ }^{2}$ Institute of Criminalistics, Prague, Czech Republic \\ Received November 24, 2014; Accepted June 1, 2015.
}

Key words: Forensic science - Forensic odontology - Dental development Demirjian's methods - Chronological age - Dental age

Abstract: The Demirjian methods to determine dental age are based on analysis of orthopantograms. The dental age estimation is based on establishing the tooth development stages. The purpose of this study was to assess the accuracy of estimation of dental age by Demirjian in the use of all of his four methods. 505 Czech healthy boys and girls aged 3 to 18 years were examined radiographically at the Department of Stomatology, Second Faculty of Medicine, Charles University in Prague. It was mentioned the factors of underlying diseases influence the accuracy of the dental age estimation. For statistical evaluation, descriptive statistics was used to compare deviations of the mean values of chronological and dental age in each age group. The resulting difference between dental age and chronological age is not significant in both genders only when using both Demirjian 7-teeth methods of 1973 and 1976. Therefore these may be most appropriately used for forensic age estimation. There are shown standard deviation differences in different countries. Demirjian's original 7-teeth method from 1973 and Demirjian's revised 4-teeth method from 1976 appear to be the best methods for calculating the dental age of healthy Czech children of both genders.

This study was supported by IGA MZCR 13351-4, 00064203, and VF 20152015041.

Mailing Address: Prof. Tat'jana Dostálová, MD., DSc., MBA, Department of Stomatology, Second Faculty of Medicine, Charles University in Prague and University Hospital Motol,V Úvalu 84, 15006 Prague 5, Czech Republic; Phone: +420 224433 101; Fax: +420 224433 120; e-mail: tatjana.dostalova@fnmotol.cz 


\section{Introduction}

With the global migration of whole families, it is nowadays important to determine the actual chronological age (CA) of people for various reasons, chiefly to determine the actual CA of children applying for asylum. There are various means of estimating the chronological age of children, for example skeletal (Greulich and Pyle, 1959; Serinelli et al., 2011). In the opinion of Finnish authors Jaasaari et al. (2012), dental development is less affected by environmental issues than skeletal maturation, and for this reason the determination of dental development is more accurate for the estimation of CA than of skeletal development. Therefore the dental age (DA) is preferred for the estimation of CA in children. Knowledge of dental age significantly helps in determining endocrinologic diagnoses in children and in planning of orthodontic treatments. DA can also be used to determine the CA of unidentified dead bodies (Feijóo et al., 2012b). DA determination is based on evaluation of teeth development.

There are many methods (Someda et al., 2009; Guo et al., 2012) by different authors - Mincer (Pechnikova et al., 2011), Pyle (Varkkola et al., 2011), Greulich (Santoro et al., 2012) - they differ in the evaluation criteria. Demirjian et al. (1973) methods are the most commonly used worldwide; they evaluate orthopantomograms (OPG) (Figure 1) and development of the evaluated teeth based on eight stages $\mathrm{A}-\mathrm{H}$ of mineralized dental tissues and closure of the apex (Figure 2). Development is calculated based on a score given to each tooth. The dental maturity score is the sum of the individual tooth scores and is subsequently converted into dental age.

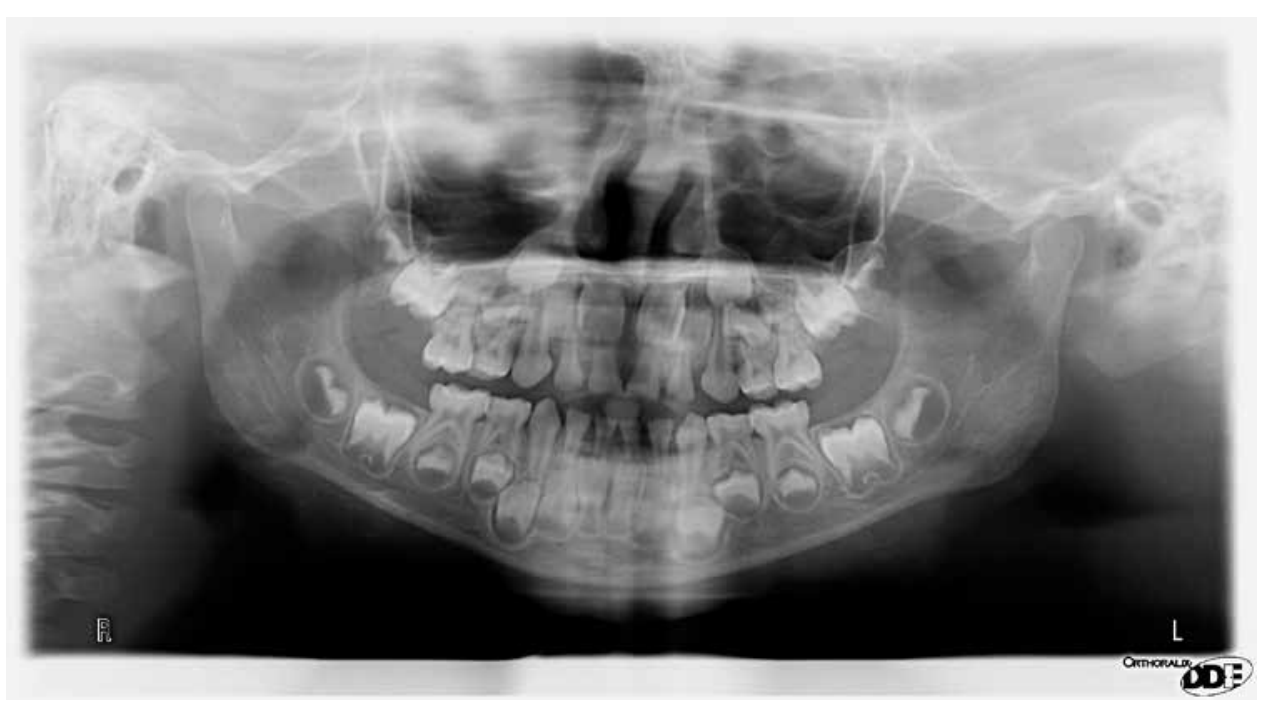

Figure 1 - Orthopantomogram - mixed dentition. 


\begin{tabular}{|c|c|c|c|c|c|c|c|c|}
\hline & A & B & C & $\mathrm{D}$ & $\mathrm{E}$ & $F$ & G & $\mathrm{H}$ \\
\hline Molars & & & & & & & & \\
\hline Bicuspid & م & $(\$)$ & $\Leftrightarrow$ & $\Leftrightarrow$ & 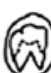 & & & \\
\hline Canines & $x$ & $\mathrm{x}$ & $\infty$ & $a$ & & & & \\
\hline Incisors & $x$ & $\mathbf{x}$ & ก & $\theta$ & $a$ & $\alpha V$ & & \\
\hline
\end{tabular}

Figure 2 - Scheme representing the classification of 8 developmental stages (Demirjian et al., 1973; Demirjian and Goldstein, 1976).

Demirjian et al. (1973) carried out a study of somatic, mental and sexual indicators of maturity on a representative sample of the French-Canadian population at the Montreal Human Growth Research Center. They came to conclusion that development of permanent dentition appeared to be the most stable process with regard to the determination of CA (Rozkovcova et al., 2012).

If necessary, in the absence of the left tooth, according to Demirjian et al. (1973), the values of the corresponding tooth on the right can be substituted for the values of the left tooth. Separate evaluations were made for groups of girls and boys, as there is, according to Demirjian et al. (1973), a difference in tooth development. Four various methods have been developed. The first and oldest of the four variants - developed in 1973, is the original method and assesses the dental maturity of the seven lower left permanent teeth. As the degree of symmetry between teeth on the left and right side is known, Demirjian et al. (1973) decided to only use one side, the lower left side. Only when a permanent tooth is missing on the left lower side (extracted, not based) can the corresponding tooth on the other, lower right side be evaluated as a substitute. The next three variants (Demirjian and Goldstein, 1976) from 1976 were modifications of Demirjian et al. (1973). In two cases Demirjian and Goldstein (1976) use only the mature stages of four permanent teeth on the lower left: in the first case M2, M1, PM2, PM1 and in the second M2, PM2, PM1, I1. We reviewed all types of assessment by Demirjian for Czech children and selected the type of rating with the smallest difference and its standard deviation (SD), expressed by certain divergences between CA and DA.

The aim of our study is to determine these differences and SD in Czech children of Caucasian population and to find out which of the four Demirjian methods (Demirjian et al., 1973; Demirjian and Goldstein, 1976) is the most suitable. 


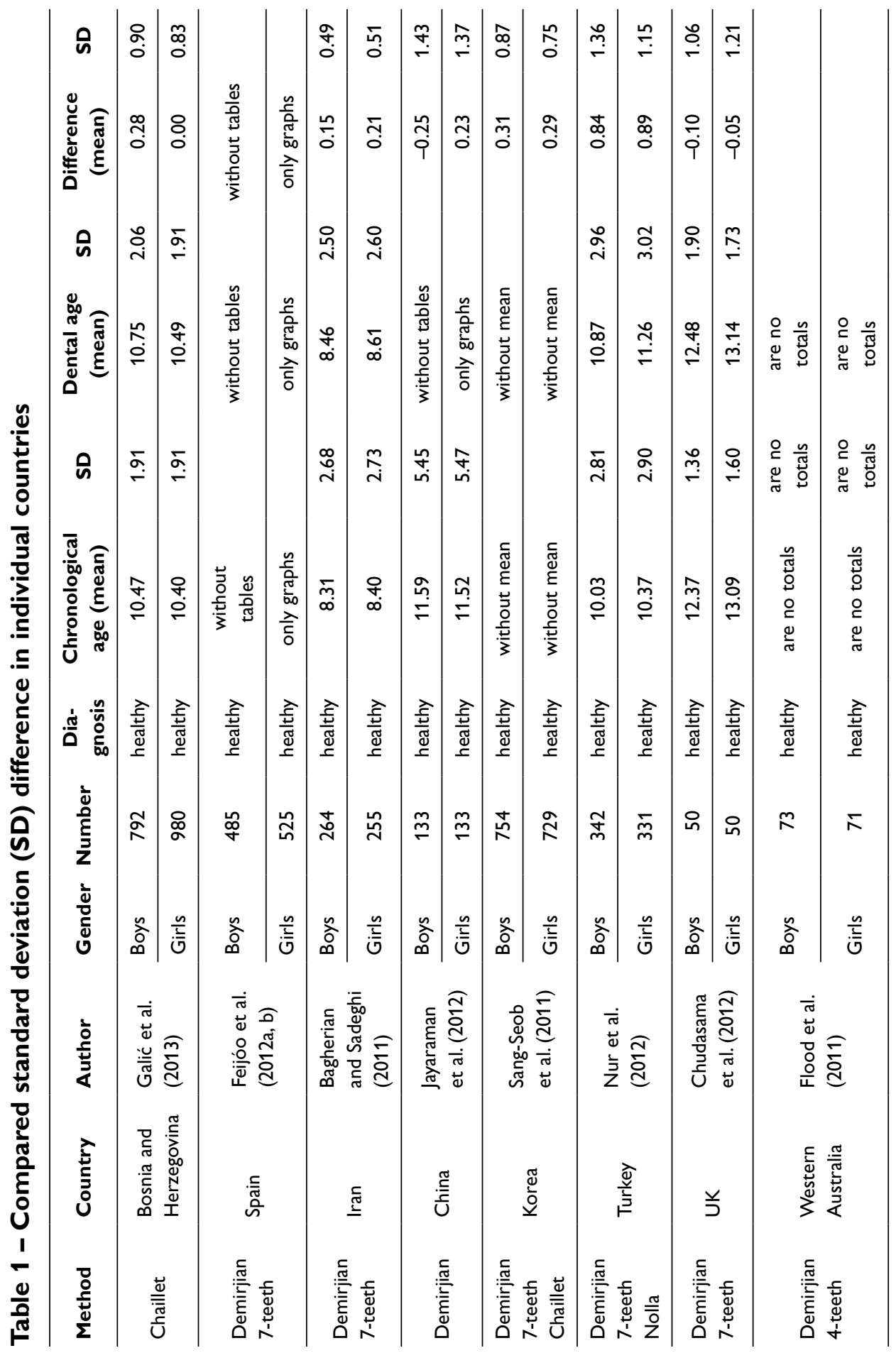




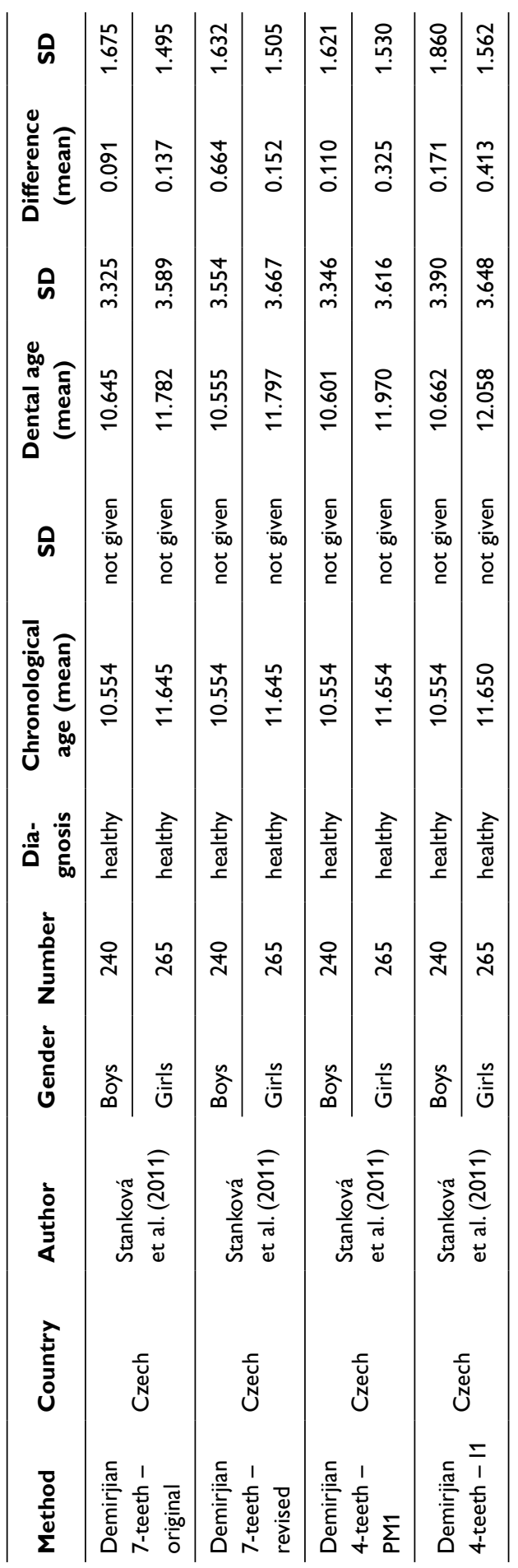


The distribution of the values detected by all four Demirjian methods for age groups, number of children and dental age and chronological age was calculated in years and months; data for SD, differences in SD and paired p-tests were used. Statistically insignificant deviations were found using Demirjian original 7-teeth method from 1973 (Demirjian et al., 1973) and the Demirjian revised 7-teeth method from 1976 (Demirjian and Goldstein, 1976). Indicators monitored in different countries according to statements in literature were also compared. A comparison of the SD of children in other countries was further made according to results reported in literature (Table 1). Several systemic diseases in children, published in the literature were neurofibromatosis (Jaasaari et al., 2012), velocardiofacial syndrome (VCFS) (Heliovaara et al., 2011).

\section{Material and Methods}

505 OPGs of children (240 boys and 265 girls) aged 3-18 years were made in the years 2011 and 2012 (Table 2). All OPGs were made using the same X-ray device (Gender Orthoralix 9 200. KaVo Dental - Gendex Imaging, Italy), excluding an error in the quality of each image. Children were commonly treated at our clinic (Department of Stomatology, Second Faculty of Medicine, Charles University in Prague), rated $\mathrm{X}$-rays were made for our treated patients - they were indicated for a reason of a dental disease. No X-ray was made only for the purpose of this study. Indications for $\mathrm{X}$-ray examinations were diagnostics and monitoring of the treatment of pathological status. All children belonged to the Caucasian population,

Table 2 - Total number of patients at each age

\begin{tabular}{lccc}
\hline Age & Boys & Girls & Total \\
\hline $2-3$ & 2 & 2 & 4 \\
4 & 1 & 4 & 5 \\
5 & 12 & 7 & 19 \\
6 & 13 & 11 & 24 \\
7 & 29 & 23 & 52 \\
8 & 40 & 18 & 58 \\
9 & 22 & 29 & 51 \\
10 & 23 & 21 & 44 \\
11 & 17 & 25 & 42 \\
12 & 19 & 23 & 42 \\
13 & 12 & 23 & 35 \\
14 & 20 & 21 & 41 \\
15 & 11 & 23 & 34 \\
16 & 10 & 21 & 31 \\
17 & 7 & 9 & 16 \\
18 & 2 & 5 & 7 \\
\hline Total & 240 & 265 & 505 \\
\hline
\end{tabular}

Ginzelová K.; Dostálová T.; Eliášová H.; Vinšů A.; Buček A.; Bučková M. 
Table 3 - Detected values for all four Demirjian methods - boys

\begin{tabular}{|c|c|c|c|c|c|c|c|}
\hline Age group & Number & $\begin{array}{l}\text { Chronolo- } \\
\text { gical age }\end{array}$ & $\begin{array}{c}1973- \\
7 \text { dental age }\end{array}$ & SD & Difference & SD & $\mathrm{P}$ \\
\hline $3-3.99$ & 2 & 3.417 & 5.200 & 0.000 & 1.783 & 0.000 & - \\
\hline $4-4.99$ & 1 & 4.667 & 5.800 & - & 1.133 & - & - \\
\hline $5-5.99$ & 12 & 5.569 & 6.575 & 0.427 & 1.006 & 0.442 & 0.000 \\
\hline $6-6.99$ & 13 & 6.526 & 7.654 & 0.602 & 1.128 & 0.684 & 0.000 \\
\hline $7-7.99$ & 29 & 7.523 & 8.041 & 0.652 & 0.518 & 0.600 & 0.000 \\
\hline 8-8.99 & 40 & 8.471 & 8.143 & 1.075 & -0.328 & 1.034 & 0.052 \\
\hline $9-9.99$ & 22 & 9.303 & 9.191 & 0.919 & -0.112 & 0.881 & 0.557 \\
\hline $10-10.99$ & 23 & 10.438 & 10.830 & 1.543 & 0.392 & 1.481 & 0.217 \\
\hline $11-11.99$ & 17 & 11.402 & 11.312 & 1.935 & -0.090 & 1.768 & 0.836 \\
\hline $12-12.99$ & 19 & 12.412 & 12.316 & 2.447 & -0.096 & 2.555 & 0.871 \\
\hline $13-13.99$ & 12 & 13.340 & 14.158 & 1.975 & 0.818 & 1.820 & 0.148 \\
\hline $14-14.99$ & 20 & 14.421 & 14.855 & 1.722 & 0.434 & 1.685 & 0.264 \\
\hline $15-15.99$ & 11 & 15.386 & 15.291 & 2.352 & -0.095 & 2.404 & 0.898 \\
\hline $16-16.99$ & 10 & 16.342 & 15.180 & 2.593 & -1.162 & 2.543 & 0.183 \\
\hline $17-17.99$ & 7 & 17.274 & 14.971 & 2.549 & -2.302 & 2.444 & 0.047 \\
\hline 18-18.99 & 2 & 18.167 & 16.000 & 0.000 & -2.167 & 0.236 & - \\
\hline Total & 240 & 10.554 & 10.645 & 3.325 & 0.091 & 1.657 & 0.394 \\
\hline Age group & Number & $\begin{array}{c}\text { Chronological } \\
\text { age }\end{array}$ & $\begin{array}{c}1976- \\
7 \text { dental age }\end{array}$ & SD & Difference & SD & $P$ \\
\hline $3-3.99$ & 2 & 3.417 & 4.900 & 0.000 & 1.483 & 0.000 & - \\
\hline $4-4.99$ & 1 & 4.667 & 5.300 & - & 0.633 & - & - \\
\hline $5-5.99$ & 12 & 5.569 & 6.217 & 0.473 & 0.647 & 0.508 & 0.001 \\
\hline $6-6.99$ & 13 & 6.526 & 7.523 & 0.763 & 0.997 & 0.812 & 0.001 \\
\hline $7-7.99$ & 29 & 7.523 & 7.997 & 0.796 & 0.474 & 0.715 & 0.001 \\
\hline 8-8.99 & 40 & 8.471 & 8.145 & 1.114 & -0.326 & 1.068 & 0.061 \\
\hline 9-9.99 & 22 & 9.303 & 9.286 & 0.937 & -0.017 & 0.899 & 0.931 \\
\hline $10-10.99$ & 23 & 10.438 & 10.939 & 1.515 & 0.501 & 1.451 & 0.112 \\
\hline $11-11.99$ & 17 & 11.402 & 11.294 & 1.822 & -0.108 & 1.674 & 0.794 \\
\hline $12-12.99$ & 19 & 12.412 & 12.226 & 2.413 & -0.186 & 2.532 & 0.753 \\
\hline $13-13.99$ & 12 & 13.340 & 14.267 & 1.942 & 0.926 & 1.798 & 0.102 \\
\hline $14-14.99$ & 20 & 14.421 & 14.525 & 1.852 & 0.104 & 1.830 & 0.802 \\
\hline $15-15.99$ & 11 & 15.386 & 15.255 & 2.088 & -0.132 & 2.144 & 0.843 \\
\hline $16-16.99$ & 10 & 16.342 & 15.180 & 2.593 & -1.162 & 2.543 & 0.183 \\
\hline $17-17.99$ & 7 & 17.274 & 15.100 & 2.381 & -2.174 & 2.269 & 0.044 \\
\hline $18-18.99$ & 2 & 18.167 & 16.000 & 0.000 & -2.167 & 0.236 & - \\
\hline Total & 240 & 10.554 & 10.555 & 3.354 & 0.064 & 1.632 & 0.652 \\
\hline
\end{tabular}

were healthy, without congenital anomalies and systemic diseases, were born on time, without significant deformity of lower left teeth, and with no significant differences in their individual social backgrounds.

Each X-ray examination is accompanied by ionizing radiation, which is dependent on the type of $X$-ray apparatus. Parameters of ionizing radiation are minimized 


\begin{tabular}{|c|c|c|c|c|c|c|c|}
\hline Age group & Number & $\begin{array}{c}\text { Chronological } \\
\text { age }\end{array}$ & $\begin{array}{l}\text { M2, M1, PM2, } \\
\text { PM1 dental age }\end{array}$ & SD & Difference & SD & $P$ \\
\hline $3-3.99$ & 2 & 3.417 & 4.900 & 0.000 & 1.483 & 0.000 & - \\
\hline $4-4.99$ & 1 & 4.667 & 4.900 & - & 0.233 & - & - \\
\hline $5-5.99$ & 12 & 5.569 & 6.208 & 0.472 & 0.639 & 0.393 & 0.000 \\
\hline $6-6.99$ & 13 & 6.526 & 7.623 & 0.867 & 1.097 & 0.882 & 0.001 \\
\hline 7-7.99 & 29 & 7.523 & 8.083 & 1.019 & 0.560 & 0.949 & 0.004 \\
\hline $8-8.99$ & 40 & 8.471 & 8.225 & 1.270 & -0.246 & 1.227 & 0.213 \\
\hline $9-9.99$ & 22 & 9.303 & 9.550 & 0.916 & 0.247 & 0.904 & 0.214 \\
\hline $10-10.99$ & 23 & 10.438 & 10.839 & 1.306 & 0.401 & 1.246 & 0.137 \\
\hline $11-11.99$ & 17 & 11.402 & 11.312 & 1.670 & -0.090 & 1.526 & 0.811 \\
\hline $12-12.99$ & 19 & 12.412 & 12.068 & 2.646 & -0.344 & 2.758 & 0.594 \\
\hline $13-13.99$ & 12 & 13.340 & 14.342 & 1.867 & 1.001 & 1.723 & 0.069 \\
\hline $14-14.99$ & 20 & 14.421 & 14.820 & 1.342 & 0.399 & 1.362 & 0.206 \\
\hline $15-15.99$ & 11 & 15.386 & 15.036 & 2.550 & -0.350 & 2.622 & 0.667 \\
\hline $16-16.99$ & 10 & 16.342 & 15.080 & 2.593 & -1.262 & 2.543 & 0.151 \\
\hline $17-17.99$ & 7 & 17.274 & 15.386 & 0.925 & -1.888 & 0.912 & 0.002 \\
\hline 18-18.99 & 2 & 18.167 & 15.900 & 0.000 & -2.267 & 0.236 & - \\
\hline Total & 240 & 10.554 & 10.601 & 3.346 & 0.110 & 1.621 & 0.343 \\
\hline Age group & Number & $\begin{array}{c}\text { Chronological } \\
\text { age }\end{array}$ & $\begin{array}{l}\text { M2, PM2, PM1, } \\
\text { I1 dental age }\end{array}$ & SD & Difference & SD & $\mathrm{P}$ \\
\hline $3-3.99$ & 2 & 3.417 & 5.700 & 0.000 & 2.283 & 0.000 & - \\
\hline $4-4.99$ & 1 & 4.667 & 6.000 & - & 1.333 & - & - \\
\hline $5-5.99$ & 12 & 5.569 & 6.242 & 0.547 & 0.672 & 0.503 & 0.001 \\
\hline $6-6.99$ & 13 & 6.526 & 7.762 & 0.985 & 1.236 & 0.951 & 0.001 \\
\hline 7-7.99 & 29 & 7.523 & 8.121 & 1.215 & 0.598 & 1.151 & 0.009 \\
\hline 8-8.99 & 40 & 8.471 & 8.305 & 1.290 & -0.166 & 1.248 & 0.406 \\
\hline $9-9.99$ & 22 & 9.303 & 9.573 & 1.462 & 0.270 & 1.452 & 0.393 \\
\hline $10-10.99$ & 23 & 10.438 & 11.030 & 1.662 & 0.592 & 1.605 & 0.091 \\
\hline $11-11.99$ & 17 & 11.402 & 11.429 & 2.397 & 0.027 & 2.315 & 0.962 \\
\hline $12-12.99$ & 19 & 12.412 & 11.963 & 2.623 & -0.449 & 2.731 & 0.483 \\
\hline $13-13.99$ & 12 & 13.340 & 13.742 & 2.679 & 0.401 & 2.567 & 0.599 \\
\hline $14-14.99$ & 20 & 14.421 & 14.820 & 1.595 & 0.399 & 1.670 & 0.298 \\
\hline $15-15.99$ & 11 & 15.386 & 15.673 & 0.754 & 0.286 & 0.950 & 0.341 \\
\hline $16-16.99$ & 10 & 16.342 & 14.710 & 3.763 & -1.632 & 3.710 & 0.198 \\
\hline $17-17.99$ & 7 & 17.274 & 15.543 & 0.945 & -1.731 & 0.847 & 0.002 \\
\hline $18-18.99$ & 2 & 18.167 & 15.900 & 0.000 & -2.267 & 0.236 & - \\
\hline Total & 240 & 10.554 & 10.662 & 3.390 & 0.171 & 1.860 & 0.220 \\
\hline
\end{tabular}

if the device that can be calibrated for X-ray examinations of children is used. Success of an X-ray examination of small children depends on the degree of cooperation of the child as well as on the cooperation between the child and the radiologist. There are indications, when it is useful to try to make a radiograph in infants not only for forensic reasons, but also to specify the diagnosis (e.g. fractures of the jaw bones). 
Table 4 - Detected values for all four Demirjian methods - girls

\begin{tabular}{|c|c|c|c|c|c|c|c|}
\hline Age group & Number & $\begin{array}{l}\text { Chronological } \\
\text { age }\end{array}$ & $\begin{array}{c}1973- \\
7 \text { dental age }\end{array}$ & SD & Difference & SD & $\mathrm{P}$ \\
\hline $3-3.99$ & 2 & 3.708 & 4.500 & 1.414 & 0.792 & 1.473 & - \\
\hline 4-4.99 & 4 & 4.604 & 4.875 & 1.190 & 0.271 & 1.400 & 0.725 \\
\hline $5-5.99$ & 7 & 5.464 & 5.971 & 1.535 & 0.507 & 1.614 & 0.438 \\
\hline $6-6.99$ & 11 & 6.379 & 7.273 & 0.403 & 0.894 & 0.489 & 0.000 \\
\hline 7-7.99 & 23 & 7.489 & 7.539 & 0.556 & 0.050 & 0.514 & 0.646 \\
\hline $8-8.99$ & 18 & 8.481 & 8.561 & 1.099 & 0.080 & 1.046 & 0.751 \\
\hline 9-9.99 & 29 & 9.434 & 9.269 & 1.215 & -0.165 & 1.149 & 0.446 \\
\hline $10-10.99$ & 21 & 10.282 & 10.157 & 1.304 & -0.125 & 1.254 & 0.654 \\
\hline $11-11.99$ & 25 & 11.517 & 11.976 & 1.871 & 0.459 & 1.879 & 0.233 \\
\hline $12-12.99$ & 23 & 12.402 & 12.835 & 1.786 & 0.433 & 1.767 & 0.253 \\
\hline $13-13.99$ & 23 & 13.482 & 14.509 & 1.295 & 1.027 & 1.243 & 0.001 \\
\hline $14-14.99$ & 21 & 14.492 & 15.662 & 1.266 & 1.170 & 1.309 & 0.001 \\
\hline $15-15.99$ & 23 & 15.518 & 15.665 & 1.056 & 0.147 & 1.020 & 0.496 \\
\hline $16-16.99$ & 21 & 16.417 & 15.748 & 0.890 & -0.669 & 0.932 & 0.004 \\
\hline $17-17.99$ & 9 & 17.361 & 14.900 & 2.479 & -2.461 & 2.395 & 0.015 \\
\hline $18-18.99$ & 5 & 18.350 & 16.000 & 0.000 & -2.350 & 0.341 & 0.000 \\
\hline Total & 265 & 11.645 & 11.782 & 3.589 & 0.137 & 1.495 & 0.137 \\
\hline Age group & Number & $\begin{array}{c}\text { Chronological } \\
\text { age }\end{array}$ & $\begin{array}{c}1976-7 \\
\text { dental age }\end{array}$ & SD & Difference & SD & $P$ \\
\hline $3-3.99$ & 2 & 3.708 & 4.450 & 1.061 & 0.742 & 1.120 & - \\
\hline $4-4.99$ & 4 & 4.604 & 4.675 & 0.981 & 0.071 & 1.193 & 0.913 \\
\hline $5-5.99$ & 7 & 5.464 & 5.714 & 1.489 & 0.250 & 1.572 & 0.689 \\
\hline $6-6.99$ & 11 & 6.379 & 6.909 & 0.611 & 0.530 & 0.673 & 0.026 \\
\hline $7-7.99$ & 23 & 7.489 & 7.317 & 0.693 & -0.172 & 0.636 & 0.209 \\
\hline $8-8.99$ & 18 & 8.481 & 8.539 & 1.207 & 0.057 & 1.151 & 0.835 \\
\hline 9-9.99 & 29 & 9.434 & 9.300 & 1.220 & -0.134 & 1.161 & 0.540 \\
\hline $10-10.99$ & 21 & 10.282 & 10.219 & 1.470 & -0.063 & 1.414 & 0.841 \\
\hline $11-11.99$ & 25 & 11.517 & 12.072 & 1.975 & 0.555 & 1.974 & 0.172 \\
\hline $12-12.99$ & 23 & 12.402 & 13.030 & 1.680 & 0.628 & 1.654 & 0.082 \\
\hline $13-13.99$ & 23 & 13.482 & 14.691 & 1.244 & 1.209 & 1.219 & 0.000 \\
\hline $14-14.99$ & 21 & 14.492 & 15.700 & 1.166 & 1.208 & 1.212 & 0.000 \\
\hline $15-15.99$ & 23 & 15.518 & 15.700 & 1.078 & 0.182 & 1.043 & 0.412 \\
\hline $16-16.99$ & 21 & 16.417 & 15.776 & 0.728 & -0.640 & 0.767 & 0.001 \\
\hline $17-17.99$ & 9 & 17.361 & 14.967 & 2.389 & -2.394 & 2.300 & 0.014 \\
\hline $18-18.99$ & 5 & 18.350 & 16.000 & 0.000 & -2.350 & 0.341 & 0.000 \\
\hline Total & 265 & 11.645 & 11.797 & 3.677 & 0.152 & 1.505 & 0.102 \\
\hline
\end{tabular}

CA was determined from the date of birth and the date taken from the OPG. CA was calculated in terms of years and months. There are 16 age groups between the ages of 3-18.99. The results for lower-left permanent teeth, except the third molar - from the first appearance of erupted teeth and tooth germs - were evaluated in terms of their development in eight stages, $\mathrm{A}-\mathrm{H}$, of mineralized dental tissues and the closure of the apex using Demirjian "Developmental stages of the 


\begin{tabular}{|c|c|c|c|c|c|c|c|}
\hline Age group & Number & $\begin{array}{c}\text { Chronological } \\
\text { age }\end{array}$ & $\begin{array}{l}\text { M2, M1, PM2, } \\
\text { PM1 dental age }\end{array}$ & SD & Difference & SD & $P$ \\
\hline $3-3.99$ & 2 & 3.708 & 4.900 & 0.141 & 1.192 & 0.200 & - \\
\hline $4-4.99$ & 4 & 4.604 & 4.850 & 1.310 & 0.246 & 1.527 & 0.769 \\
\hline $5-5.99$ & 7 & 5.464 & 6.043 & 1.518 & 0.579 & 1.590 & 0.373 \\
\hline $6-6.99$ & 11 & 6.379 & 7.236 & 0.709 & 0.858 & 0.697 & 0.002 \\
\hline 7-7.99 & 23 & 7.489 & 7.478 & 0.926 & -0.011 & 0.858 & 0.952 \\
\hline $8-8.99$ & 18 & 8.481 & 8.928 & 1.278 & 0.446 & 1.233 & 0.143 \\
\hline $9-9.99$ & 29 & 9.434 & 9.548 & 1.214 & 0.114 & 1.161 & 0.600 \\
\hline $10-10.99$ & 21 & 10.282 & 10.352 & 1.806 & 0.071 & 1.759 & 0.856 \\
\hline $11-11.99$ & 25 & 11.517 & 12.172 & 2.282 & 0.655 & 2.307 & 0.168 \\
\hline $12-12.99$ & 23 & 12.402 & 13.252 & 1.932 & 0.850 & 1.892 & 0.042 \\
\hline $13-13.99$ & 23 & 13.482 & 15.004 & 1.085 & 1.522 & 1.066 & 0.000 \\
\hline $14-14.99$ & 21 & 14.492 & 15.590 & 1.191 & 1.098 & 1.236 & 0.001 \\
\hline $15-15.99$ & 23 & 15.518 & 15.817 & 0.292 & 0.299 & 0.357 & 0.001 \\
\hline $16-16.99$ & 21 & 16.417 & 15.671 & 0.723 & -0.745 & 0.751 & 0.000 \\
\hline $17-17.99$ & 9 & 17.361 & 15.556 & 0.720 & -1.806 & 0.709 & 0.000 \\
\hline 18-18.99 & 5 & 18.350 & 15.900 & 0.000 & -2.450 & 0.341 & 0.000 \\
\hline Total & 265 & 11.645 & 11.970 & 3.616 & 0.325 & 1.530 & 0.001 \\
\hline Age group & Number & $\begin{array}{c}\text { Chronological } \\
\text { age }\end{array}$ & $\begin{array}{l}\text { M2, PM2, PM1, } \\
\text { I1 dental age }\end{array}$ & SD & Difference & SD & $P$ \\
\hline $3-3.99$ & 2 & 3.708 & 5.100 & 0.283 & 1.392 & 0.342 & - \\
\hline $4-4.99$ & 4 & 4.604 & 5.350 & 0.742 & 0.746 & 0.856 & 0.180 \\
\hline $5-5.99$ & 7 & 5.464 & 6.171 & 1.230 & 0.707 & 1.335 & 0.211 \\
\hline $6-6.99$ & 11 & 6.379 & 7.073 & 0.714 & 0.694 & 0.735 & 0.011 \\
\hline 7-7.99 & 23 & 7.489 & 7.500 & 0.915 & 0.011 & 0.844 & 0.951 \\
\hline 8-8.99 & 18 & 8.481 & 8.872 & 1.383 & 0.391 & 1.340 & 0.233 \\
\hline $9-9.99$ & 29 & 9.434 & 9.807 & 1.346 & 0.373 & 1.270 & 0.125 \\
\hline $10-10.99$ & 21 & 10.282 & 10.548 & 1.926 & 0.266 & 1.859 & 0.520 \\
\hline $11-11.99$ & 25 & 11.517 & 12.232 & 2.178 & 0.715 & 2.199 & 0.117 \\
\hline $12-12.99$ & 23 & 12.402 & 13.091 & 2.399 & 0.689 & 2.347 & 0.173 \\
\hline $13-13.99$ & 23 & 13.482 & 15.117 & 1.152 & 1.636 & 1.138 & 0.000 \\
\hline 14-14.99 & 21 & 14.492 & 15.676 & 1.255 & 1.184 & 1.299 & 0.000 \\
\hline $15-15.99$ & 23 & 15.518 & 15.909 & 0.303 & 0.391 & 0.364 & 0.000 \\
\hline $16-16.99$ & 21 & 16.417 & 15.867 & 0.432 & -0.550 & 0.482 & 0.000 \\
\hline 17-17.99 & 9 & 17.361 & 15.744 & 0.767 & -1.617 & 0.696 & 0.000 \\
\hline 18-18.99 & 5 & 18.350 & 16.000 & 0.000 & -2.350 & 0.341 & 0.000 \\
\hline Total & 265 & 11.645 & 12.058 & 3.648 & 0.413 & 1.562 & 0.000 \\
\hline
\end{tabular}

permanent dentition" table (Figure 1) (Demirjian et al., 1973; Tanner et al., 1997). The position of the lower-left teeth was marked in the same order as in the Demirjian methods: second molar M2, first molar M1, second premolar PM2, first premolar PM1, canine C, lateral incisor 12, central incisor 11 (Demirjian et al., 1973). The M1 mandible left tooth was missing in only four children extracted due to destruction and the corresponding right mandible permanent teeth were therefore evaluated instead. Dental age was established using all four types of evaluation: 
Demirjian original 7-teeth 1973 method (Demirjian et al., 1973), the revised 1976 teeth method and the two 1976 4-teeth methods (Demirjian and Goldstein, 1976). CA, DA and SD difference by age category, broken down by gender, is shown in Table 3 for boys and Table 4 for girls, all of which were evaluated using all Demirjian methods. The relationship between dental maturity score and CA is expressed and plotted in the percentile graphs (Demirjian et al., 1973) (Figure 3). The dental maturity score is the sum of individual teeth scores. Standard tables (Demirjian et al., 1973) were used to calculate dental age from the dental maturity score. All four Demirjian methods were compared and statistically evaluated for average chronological age and its relationship to dental age. Deviation from SD was evaluated using the paired $\mathrm{p}$-test.

A comparison with deviations in various countries was made against results reported in literature (Table 1 ).

All observations were carried out in accordance with the Helsinki Declaration. Ethical approval for the study was obtained from the Ethics Committee of the Second Faculty of Medicine, Charles University in Prague (Rozkovcova et al., 2012).

DA was calculated using the score determined using tables of different systems: Demirjian 7-teeth 1973, Demirjian 7-teeth 1976, Demirjian 4-teeth M2, M1, PM2, PM1, Demirjian 4-teeth M2, PM2, PM1, I1 (Demirjian et al., 1973; Demirjian and Goldstein, 1976), converted to dental age.

Descriptive statistics (mean, SD) were used for statistical evaluation and paired $t$-test was used to compare deviations of mean values for CA and DA in each age group.

The evaluation was carried out with a significance level of 0.05 .

Data was processed using statistical functions of MS Excel 2013 and the Data Analysis Toolpack (MS Excel 2013) analytical add-on.

\section{Results}

Distribution of the values detected by all four Demirjian methods (Demirjian et al., 1973; Demirjian and Goldstein, 1976) for age groups, number of children and DA and CA was calculated in years and months; data for SD, differences in SD and paired $p$-tests are presented in Table 3 for boys and Table 4 for girls. These Tables give a comprehensive overview of the observed values. The listed values are further processed in the following Tables.

\section{Chronological age (CA)}

The mean CA for boys was 10.552 years and 11.645 years for girls.

Dental age (DA)

Estimated DA can be overestimated or underestimated (Demirjian et al., 1973; Sang-Seob et al., 2011) and the mean deviation of DA from CA varies (Table 5). DA is overestimated by all Demirjian's methods in girls, and by two methods in 
Table 5 - Dental age: Number of age groups underestimated and overestimated

\begin{tabular}{|c|c|c|c|c|c|}
\hline \multirow{2}{*}{$\begin{array}{l}\text { Demirjian } \\
\text { methods }\end{array}$} & \multicolumn{2}{|c|}{ Total number } & \multirow{2}{*}{$\begin{array}{l}\text { Difference } \\
\text { (mean) }\end{array}$} & \multirow[t]{2}{*}{ SD } & \multirow[t]{2}{*}{$P$} \\
\hline & Overestimated & Underestimated & & & \\
\hline \multicolumn{6}{|c|}{ Boys: total of 16 age groups } \\
\hline 7-teeth 1973 & 8 & 8 & 0.091 & 1.657 & 0.394 \\
\hline 7-teeth 1976 & 8 & 8 & 0.064 & 1.632 & 0.652 \\
\hline 4-teeth PM1 & 7 & 9 & 0.110 & 1.621 & 0.343 \\
\hline 4-teeth I1 & 5 & 11 & 0.171 & 1.860 & 0.220 \\
\hline \multicolumn{6}{|c|}{ Girls: total of 16 age groups } \\
\hline 7-teeth 1973 & 11 & 5 & 0.137 & 1.495 & 0.137 \\
\hline 7-teeth 1976 & 10 & 6 & 0.152 & 1.505 & 0.102 \\
\hline 4-teeth PM1 & 12 & 4 & 0.325 & 1.530 & $0.001 *$ \\
\hline 4-teeth I1 & 13 & 3 & 0.413 & 1.562 & $0.000^{*}$ \\
\hline
\end{tabular}

*significant

boys - Demirjian 4-teeth PM1 and Demirjian 4-teeth I1 (Demirjian and Goldstein, 1976). Results are the same in the two residual methods. SD occurs with only two methods in girls - Demirjian 4-teeth PM1 and Demirjian 4-teeth I1 (Demirjian and Goldstein, 1976).

\section{Difference DA-CA}

Accuracy of each method is expressed by the difference between DA and CA. SD and the paired $t$-test were monitored. A comparison was made of results for boys and girls. There were varying degrees of difference for each age group (Tables 3 and 4) and varying sizes (Table 6).

\section{Percentile chart}

The percentile chart clearly shows that the relationship between dental maturity scores and children age is not linear (Figure 3). This can be more easily observed in the graph than in the tables.

\section{Different countries}

Indicators monitored in different countries according to statements in literature are compared in Table 1.

\section{Discussion}

All four Demirjian methods were used, being practical and simple. Demirjian methods were based on analysis of OPG (Demirjian et al., 1973; Demirjian and Goldstein, 1976). DA estimation was based on establishment of 8 teeth development stages; assessment is based on evaluation of the OPG. It was 
Table 6 - The greatest and the least values of difference

\begin{tabular}{|c|c|c|c|c|c|c|}
\hline & \multicolumn{2}{|c|}{ The greatest difference } & \multicolumn{2}{|c|}{ The least difference } & \multicolumn{2}{|c|}{ Total } \\
\hline & Boys & Girls & Boys & Girls & Boys & Girls \\
\hline \multicolumn{7}{|c|}{ Demirjian original method 1973 - 7-teeth } \\
\hline Years & $3-4$ & $14-15$ & $17-18$ & 17-18 & - & - \\
\hline Difference & 1.783 & 1.170 & -2.302 & -2.461 & 0.091 & 0.137 \\
\hline SD & - & 1.309 & 2.444 & 2.395 & 1.657 & 1.495 \\
\hline$P$ & - & $0.001 *$ & $0.047^{*}$ & $0.015^{*}$ & 0.394 & 0.137 \\
\hline \multicolumn{7}{|c|}{ Demirjian revised method 1976 - 7-teeth } \\
\hline Years & $3-4$ & $13-14$ & $17-18$ & 17-18 & - & - \\
\hline Difference & 1.483 & 1.209 & -2.174 & -2.394 & 0.064 & 0.152 \\
\hline SD & - & 1.219 & 2.269 & 2.300 & 1.632 & 1.505 \\
\hline$P$ & - & $0.000 *$ & $0.044^{*}$ & $0.014 *$ & 0.659 & 0.102 \\
\hline \multicolumn{7}{|c|}{ Demirjian method 1976 - 4-teeth (M2, M1, PM2, PM1) } \\
\hline & Boys & Girls & $\begin{array}{l}\text { Lesser } \\
\text { difference }\end{array}$ & Boys & Girls & Total \\
\hline Years & $3-4$ & $13-14$ & 18-19 & $18-19$ & - & - \\
\hline Difference & 1.483 & 1.552 & -2.267 & -2.450 & 0.064 & 0.325 \\
\hline SD & 0.000 & 1.066 & 0.236 & 0.341 & 1.632 & 1.530 \\
\hline$P$ & - & $0.000 *$ & - & $0.000 *$ & 0.652 & $0.001 *$ \\
\hline \multicolumn{7}{|c|}{ Demirjian method 1976 - 4-teeth (M2, PM2, PM1, I1) } \\
\hline Years & $3-4$ & $13-14$ & 18-19 & $18-19$ & - & - \\
\hline Difference & 2.283 & 1.636 & -2.267 & -2.350 & 0.171 & 0.413 \\
\hline SD & 0.000 & 1.138 & 0.236 & 0.341 & 1.860 & 1.562 \\
\hline $\mathrm{P}$ & - & $0.000 *$ & - & 0.000 & 0.220 & $0.000 *$ \\
\hline
\end{tabular}

*significant; - = cannot be count (under age)

important to obtain reliable results that evaluated the OPG of the same persons in order to eliminate subjective deviations.

The mean CA was 10.552 years for boys and 11.645 years for girls. Spanish literature gives a mean age of 9.2 for both genders (Feijóo et al., 2012a).

Conversion of the dental maturity score of each subject using Demirjian standard tables for children in the two age groups from 16-18 years is not quite accurate. Demirjian evaluates children only at 16 years of age (Demirjian et al., 1973). In the Czech Republic, children are considered adolescents up to their eighteenth birthday, a fact which must be respected if the results of forensic tests are to be used. Conversion was performed according to Demirjian tables (Demirjian et al., 1973). 
Nur et al. (2012) show overestimation of estimated DA against actual CA in both genders, and our results show overestimation for all Demirjian's methods (Demirjian et al., 1973; Demirjian and Goldstein, 1976) in girls, and in two methods - Demirjian 4-teeth PM1 and Demirjian 4-teeth I1 - in boys, with the same results in the two residual methods (Table 5). There was a mean paired $t$-test difference greater than 0.05 in all four Demirjian methods for boys and in two Demirjian methods for girls - Demirjian 4-teeth PM1 and Demirjian 4-teeth 11 - no group was therefore of statistical significance; however, Demirjian 4-teeth PM1 method and Demirjian 4-teeth 11 method were significant in girls (Table 5). The different values (Tables 3 and 4) in children's teeth development according to the estimation of DA based on maturity of tooth germs (Demirjian et al., 1973) are influenced by nutrition, climate and social and economic conditions (Burt et al., 2011).

Accuracy of each method is expressed as the difference between DA and CA. Variety between DA and CA is expressed by the difference between DA and $C A$ and can have a positive or negative value (Demirjian et al., 1973) (Table 5). Accuracy of the estimated DA is expressed as the value of the SD difference. The estimated DA in our groups of children is the least accurate in the youngest and the oldest age groups; Sang-Seob et al. (2011) also indicates this. This explains the small number of 3-year-olds, and the peculiarities in the conversion of dental maturity in 16-18-year-olds.

No records at all exist of deviations from DA estimates caused by systemic diseases; children's overall health status must be taken into account.

In the event of neurofibromatosis type 1 (Jaasaari et al., 2012), dental development is more accurate for the purposes of estimating CA than skeletal development. In this case, skeletal maturation is affected, while dental development is standard. Dental maturity is never delayed in children with VCFS (Heliovaara et al., 2011).

\section{Conclusion}

Dental age estimation in children is based on the establishment of stages of tooth development. It is important to thoroughly examine, clinically and with X-ray, the real age of children from the viewpoint of pediatric dentistry and also pediatrics as a whole. Based on our results, Demirjian methods - original 7-teeth 1973 and Demirjian revised 4-teeth 1976 (Demirjian et al., 1973; Demirjian and Goldstein, 1976) - appear to be the best methods for calculating the DA of healthy Czech children of both genders. The mean of paired $t$-tests for difference between DA and $C A$ showed no statistically significant SD in either gender.

\section{References}

Bagherian, A., Sadeghi, M. (2011) Assessment of dental maturity of children aged 3.5 to 13.5 years using the Demirjian method in an Iranian population. J. Oral Sci. 53, 37-42. 
Burt, N. M., Sauer, N., Fenton, T. (2011) Testing the Demirjian and the international Demirjian dental aging methods on a mixed ancestry urban American subadult sample from Detroit, MI. J. Forensic Sci. 56, 1296-1301.

Chudasama, P. N., Roberts, G. J., Lucas, V. S. (2012) Dental age assessment (DAA): A study of a Caucasian population at the 13 year threshold. J. Forensic Leg. Med. 19, 22-28.

Demirjian, A., Goldstein, H. (1976) New systems for dental maturity based on seven and four teeth. Ann. Hum. Biol. 3, 411-421.

Demirjian, A., Goldstein, H., Tanner, J. M. (1973) A new system of dental age assessment. Hum. Biol. 45, 211-227.

Feijóo, G., Barbería, E., De Nova, J., Prieto, J. L. (2012a) Permanent teeth development in a Spanish sample. Application to dental age estimation. Forensic Sci. Int. 214, 213.e1-213.e6.

Feijóo, G., Barbería, E., De Nova, J., Prieto, J. L. (2012b) Dental age estimation in Spanish children. Forensic Sci. Int. 223, 371.e1-371.e5.

Flood, S. J., Mitchell, W. J., Oxnard, Ch. E., Turlach, B. A., McGeachie, J. (2011) To evaluate the utility of smaller sample sizes when assessing dental maturity curves for forensic age estimation. J. Forensic Sci. 56, 16041609.

Galić, I., Vodanović, M., Janković, S., Mihanović, F., Nakać, E., Prohić, S., Galić, E., Brkić, H. (2013) Dental age estimation on Bosnian-Herzegovinian children aged 6-14 years: Evaluation of Chaillet's international maturity standards. J. Forensic Leg. Med. 20, 40-45.

Greulich,W.W., Pyle, S. I. (1959) Radiographic Atlas of Skeletal Development of the Hand and Wrist. Stanford University Press, Stanford.

Guo, L., Jiayin, R., Shuping, Z., Yuanyuan, L., Na, L., Wanhong, W., Shanshan, H.W. (2012) Dental age estimation from the developmental stage of the third molars in western Chinese population. Forensic Sci. Int. 219 , 158-164.

Heliovaara, A., Rantanen, I., Arte, S. (2011) Dental development and tooth agenesis in children with velocardiofacial syndrome. Int. J. Paediatr. Dent. 21, 446-450.

Jaasaari, P., Visnapuu, V., Nystrom, M., Peltonen, S., Peltonen, J., Happonen, R. P. (2012) Dental age in patients with neurofibromatosis 1. Eur. J. Oral Sci. 120, 549-552.

Jayaraman, J., Roberts, G. J., King, N. M., Wong, H. M. (2012) Dental age assessment of southern Chinese using the United Kingdom Caucasian reference dataset. Forensic Sci. Int. 216, 68-72.

Nur, B., Kusgoz, A., Bayram, M., Celikoglu, M., Nur, M., Kayipmaz, S., Yildirim, S. (2012) Validity of Demirjian and Nolla methods for dental age estimation for northeastern Turkish children aged 5-16 years old. Med. Oral Patol. Oral Cir. Bucal 17, e871-e877.

Pechnikova, M., Gibelli, D., De Angelis, D., De Santis, F., Cattaneo, C. (2011) The blind age assessment: Applicability of Greulich and Pyle, Demirjian and Mincer aging methods to a population of unknown ethnic origin. Radiol. Med. 116, 1105-1114.

Rozkovcova, E., Dostalova, T., Markova, M., Broukal, Z. (2012) The third molar as an age marker in adolescents: new approach to age evaluation. J. Forensic Sci. 57, 1323-1328.

Sang-Seob, L., Dongjae, K., Saebomi, L., U-Young, L., Joong, S. S., Yong, W. A., Seung-Ho, H. (2011) Validity of Demirjian's and modified Demirjian's methods in age estimation for Korean juveniles and adolescents. Forensic Sci. Int. 211, 41-46.

Santoro, V., Roca, R., De Donno, A., Fiandaca, Ch., Pinto, G., Tafuri, S., Introna, F. (2012) Applicability of Greulich and Pyle and Demirjian aging methods to a sample of Italian population. Forensic Sci. Int. 221, 153.e1-153.e5.

Serinelli, S., Panetta, V., Pasqualetti, P., Marchetti, D. (2011) Accuracy of three age determination X-ray methods on the left hand-wrist: A systematic review and meta-analysis. Leg. Med. (Tokyo) 13, 120-133. 
154) Prague Medical Report / Vol. 116 (2015) No. 2, p. 139-154

Someda, H., Saka, H., Matsunaga, S., Ide, Y., Nakahara, K., Hirata, S., Hashimoto, M. (2009) Age estimation based on three-dimensional measurement of mandibular central incisors in Japanese. Forensic Sci. Int. 185, 110-114.

Stanková, M., Buček, A., Dostálová, T., Ginzelová, K., Pacáková, Z., Seydlová, M. (2011) Patients with special needs within treatment under general anesthesia - Meta-analysis. Prague Med. Rep. 112, 216-225.

Tanner, J., Oshman, D., Bahhage, F., Healy, M. (1997) Tanner-Whitehouse bone age reference values for North American children. J. Pediatr. 131, 34-40.

Varkkola, O., Ranta, H., Metsaniitty, M., Sajantila, A. (2011) Age assessment by the Greulich and Pyle method compared to other skeletal $X$-ray and dental methods in data from Finnish child victims of the Southeast Asian Tsunami. Forensic Sci. Med. Pathol. 7, 311-316. 